\title{
REHA - A Multimedia System to Learn About IT-Systems for Disabled Persons
}

\author{
O. Rienhoff, H. Wittchow \\ Institute of Medical Informatics, University \\ Marburg, Bunsenstrasse 3, 35033 Marburg, F.R.G. \\ FAX: +49-6421-28-8921
}

\begin{abstract}
In the last five years $R \& D$ activities in the field of informationtechnology-support for disabled persons have boomed. However, despite many impressive pieces of technology no breakthrough to a broad usage of this technology has happened. In this context an interdisciplinary working group at the Institute of Medical Informatics worked on the didactic- and presentation concept of Reha, a multimedia learning system, orientated towards three main target groups for information technoloy for the disabled: physicians, medical students, and patients. The concept was transformed into a demonstrator.

The program offers information and explanations about certain diseases. From there is a direct branching to the IT-aid part of the program to introduce a corresponding IT-aid that seems to be suitable.
\end{abstract}

\section{$1 \quad$ Silent Market}

In the last five years $R \& D$ activities in the field of information technology support for disabled persons have boomed. Not only that the European Union has formulated and carried out a major R\&D program (TIDE) under its telematics activities but many research institutes, hospitals, rehabilitation institutions, medium and large enterprises have invested into the sector.

However, despite many impressive pieces of technology, despite some equally famous users like Stephen Hawking, no breakthrough to a broad usage of this technology has happened. Patients and doctors often do not know about existing possibilities. As a consequence, not enough consumer requests are made to establish a regional, national or European market and consequently prices for appropriate equipment are high and distribution of products is slow and hesitating. 
The Mid Term Report to the Third Framework Program of the European Union regards the TIDE program as not too successful due to the fact that the market is extremely fragmented and that mainly small enterprises have been active in R\&D work.

The results of the booming $R \& D$ activities are presented at more and more conferences and fairs. But as long as the market stays silent no cash flow will back up the further advancement of the technology. In this case an upswing can be expected and the possible impact of the new technology for disabled persons may be limited.

In this context, the Institute of Medical Informatics at Marburg University was looking for a booster for the slowly evolving consumer market.

\section{The REHA Booster}

The medical market is primarily driven by health care providers who prescribe drugs or sometimes medical equipment. In most countries of Europe physicians play this role. To a limited degree a similar function is related to other health care professionals like nurses for home care or e.g. orthopaedic shops. Despite efforts to a more active role of patients or disabled persons the key to a more effective use of information technology for disabled persons lies in the hands of physicians and other health professionals.

However, from studies it is known that many health professionals have kept a distance to information technology. In most European countries they do not get any serious introduction into medical informatics during their medical training. A good example for this distance is the distribution of computer systems in private physicians' offices. It is only higher in those European countries which have a governmental promotion policy (e.g. the UK.). If one further realizes that the development of IT support for disabled persons is still very much in its early development it becomes immediately clear that the key prescribers of this technology do never learn about the impressive results which can be achieved already today.

Therefore, a tool is needed to effectively inform: practising doctors, medical students, other health professionals, and patients about general possibilities on the one hand and about specific products on the other.

As the development of such an information tool needs a substantive investment effort and a continuous updating activity, in 1993 a concept for a multimedia learning system was derived: REHA. 
REHA tries to utilize as many existing information sources as possible, e.g. the database Rehadat which is provided by the Institut der Deutschen Wirtschaft. Reha is also based on some of the most commonly available software tools like MS-Windows ${ }^{\circledR}$ and Toolbook ${ }^{\circledR}$. An interdisciplinary working group at the Institute at Marburg worked for six months on the didactic- and presentation concept of REHA and transformed the concept into a demonstrator which was presented at the CEBIT fair at Hannover in March 1994. The demonstrator has all key functions of the REHA concept and is exemplified for the field of IT support for persons with limited eyesight.

The curricular modules are organized in three layers. The top layer is a general layer which contains all information about REHA and how to use it. The intermediate layer contains all information modules regarding various types of disabilities and correspondingly various types of information technology to overcome shortcomings caused by the disabilities. The bottom layer is a medical technical encyclopedia which contains all specific terms which are used in the modules above.

\section{REHA - the Principal Structure}

Fundamentally, the program puts the various modules to the user's disposal as shown below.

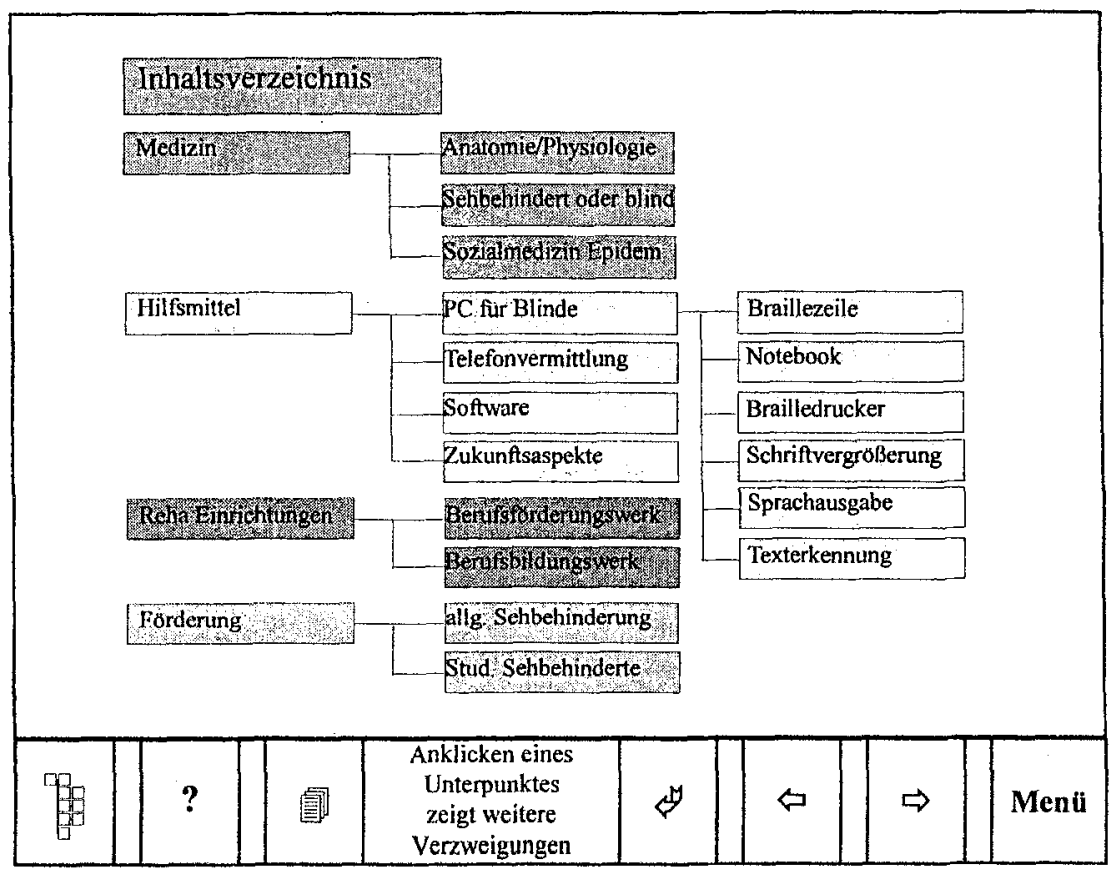


Contents of the module (translation of figure)

Medicine Some different types of disabilities are shown here including typical diagnoses and their possible etiology. They are consolidated with information and statistics being part of social medicine.

IT-aid Due to the possibilities of various different media, the user can get a very realistic demonstration of IT-based aid, whose categorisation follows the rules of the standardised ISOclassification.

Database

Some examples are made available for the learner. With the help of these examples they can practise to make searches in the database REHADAT.

Supportive Information about specialised institutions of rehabilitation and measures supportive measures are given to the user of REHA.

It is worth mentioning that no previous knowledge is expected to work with the different modules. Using the advantages of a multimedia system different media have been used whenever it seemed reasonable. In addition to text information pictures, picture sequences, demonstrations, sound and simulations (e.g. using a Braillekeyboard) are used to fulfil every type of learners' expectations as there are auditive, visual, haptic and intellectual types of learners. Information that are foreign to the subject but imperative as well, e.g. those of the sector of informatics, are explained and illustrated or shown as a demonstration to make learning come alive.

\section{The Curricular Design of REHA}

The curricular design of REHA is orientated towards its three main target groups: physicians, medical students, and patients. With respect to the target groups, different paths through the various system modules are recommended.

The program allows practising doctors two different possibilities to use it:

- $\quad$ as a learning program for the target groups: physicians, students and patients

- $\quad$ as an explanation aid within a confidential talk between a physician and a patient, during a medical consultation or treatment episode 
The access to the program will be basically different in the two cases. In the first case the access will be dominated by interest, that means the user is intrinsically motivated while, in the latter, the access will go straight up to the patient's problem.

The physician can give some information and explanations about a certain disease the patient is suffering from. The medical part of the program allows to show corresponding pages at the physician's disposal. Following, there is a direct branching to the IT-aid part of the program, so the physician may introduce an IT-aid that seems to be suitable.

It is not necessary to describe the course of access usually taken when other health professionals use REHA, because it is similar to the way described above.

To medical students the program REHA offers a new and different approach to the topic disability. Pure medical knowledge may immediately be completed by information about IT-aid. There are "buttons" on the pages of the medical part of REHA that offer a branching to corresponding pages of the IT-aid part of REHA. The buttons carry icons with a high stimulative nature suggesting sound-, picture- and video-information. As a supplement there is a "personal experience part" to the medical module: with this typical multimedia function medical students can personally experience disabilities, e.g. how patients, suffering from retinopathia pigmentosa perceive their environment. The students ought to have experienced it themselves because this possibility may lead to a more emotional affinity to the topic disability.

\section{The Didactic Layout of REHA}

The didactic layout is following the general principles of the design of multimedia systems in the teaching context (see literature). All modules follow in their layout a set of rules valid for the entire system.

\section{1 Structure of the Program}

- The program is not split into definite chapters or learning units (like in a book), so the work with the program can be organised in a very individual way.

- The program does not have to be worked through sequentially, it can be interrupted at any time. This individually adapted shortest sequences of time can be used for learning. 
- To fulfil the various expectations of the main target groups with their different background knowledge, REHA offers an encyclopedia function as a constant feature to look up unknown terms. A simple click on the unknown term inserts a spiral pad on the right part of the screen and without leaving the actual page the user gets further explanations to the searched term.

\subsection{Using the Program}

- Neither a manual nor much time is necessary to work with REHA as it is easy to handle with a mouse. Only four buttons will let you move through the program.

There are three more buttons to make the work easier: With a click on the "?" button the user can ask for on-line help which supplies extensive additional help. After a click on the "structure button" the structure of the main parts of the program are shown, details can be requested. A mark shows the actual position, there is no danger to get lost in hyper space.

A click on the "靣" - button will branch to a page that contains an index on its left part. Choosing one of the listed headwords an explanation of the headword is shown on the right part of the page. Supplementary an offer is made to branch to the corresponding page of the program which deals with the topic. This facilitates the access to the program led by headwords and is therefore very fast.

All the buttons are activated with a simple mouse-click. They have got the same function, same appearance and same position on the screen throughout the entire program.

- Within any text further information can be offered: On the screen are special areas with a short hint of the topic and a special symbol to show whether further information is available as sound, picture or video information. The information to which part of the program the module is branching is withhold from the user intentionally. The choice of the special area shall entirely be based on the interest of the user. The program supports such "free explorations". There is no necessity for the user to remember complex return routes, with the help of the " $\$$ " button the skip back to the last branching is possible.

- The layout was unified for all modules. For an easier orientation a different colour was chosen for the background of each of the four modules (medicine, ITaid, database and supportive measures). The reddish background for example automatically indicates that your position is somewhere in the medicine module.

- The headings of chapters, text information and pictures have got their fixed position on the screen. 
- Single pictures of film/audio-sequences are at least presented for six seconds. Sequences must be started by the user, they never start automatically. In this way it is avoided that two sources overlap and may thereby disturb each other. According to this the screen is presented in grey while sound information is presented. The principle is to allow concentration on one medium only.

The interface to the Rehadat database which holds actual information about products is being programmed now. For the demonstrator it was acceptable to have some dummy frames in the system to show the principle and receive user reactions.

The Rehadat interface will be the first example of interfaces to other systems. REHA can only survive if it can utilize databases which are maintained by commercial organizations.

\section{REHA - Hardware and Software Requirements}

\subsection{Software Requirements:}

- DOS 3.1 or higher

- Microsoft Windows 3.0 or higher

- ToolBook® 1.5 (Asymetrix Corporation)

\subsection{Hardware Requirements:}

The recommended minimum system configuration to run Reha and ToolBook® is:

- A personal computer with at least a $16 \mathrm{MHz} 80386 \mathrm{SX}$ processor

- 2MB RAM (4MB recommended)

- $1.4 \mathrm{MB}(31 / 2 ")$ disk drive

- A hard disk with min. 10MB of free disk space

- A monitor and graphics card for VGA or VGA Plus

- A Windows-compatible mouse

- An MCI-compatible audio subsystem, such as the SoundBlaster Pro board 


\section{Test of REHA}

The exhibition of the REHA demonstration at the CEBIT was part of a first testing phase in which physicians, medical students, and visitors of the fair were asked to comment on the system. This led to a revision of the first version in April and May 1994. REHA 1.1 has in the meantime attracted the interest of two health insurance companies in Germany after being demonstrated to them in the current version.

A major validation phase is prepared within the department to test the demonstrator, its curricular design and the handling of the system with groups of medical students from the Medical School. These tests will lead to a revised version 1.2 which also shall include the functioning interface to the actual Rehadat database. Version 1.2 will be the basis of horizontal extensions of REHA into other areas of human impairment.

\section{Literature}

SCHAUB, M. (1992). Code X: multimediales Design. DuMont Buchverlag, Köln.

EULER, D. (1992). Didaktik des computerunterstützten Lernens: praktische Gestaltung und theoretische Grundlagen. Bildung und Wissenschaft Verlag, Nürnberg.

HANNAFIN M. J. \& PECK, K. L. (1988). The Design, Development, and Evaluation of Instructional Software. Macmillan, New York.

FRIEDRICH, H. \& MANDL, H. (1990). Psychologische Aspekte autodidaktischen Lernens. In: Unterrichtswissenschaft 3, 197-218.

ISSING, L. J. (1988) Wissenserwerb mit Medien. In: H. Mandl \& H. Spada (Hrsg.): Wissenspsychologie. Psychologie VerlagsUnion, München-Weinheim.

WEIDENMANN, B. (1986). Psychologie des Lernens unit Medien. In: B. Weidenmann \& A. Krapp (Hrsg.) Pädagogische Psychologie. Psychologie VerlagsUnion, München-Weinheim. 